\title{
Propuesta, indicadores vinculados a la sostenibilidad turística en Pastaza, Ecuador
}

\section{Proposal, indicators linked to tourism sustainability in Pastaza, Ecuador}

Clímaco Geovani Espín Ortíz. ${ }^{1}$, Edison Eduardo Ruiz. ${ }^{2}$, Julian Leoncio Rodríguez Rodríguez. ${ }^{3}$

\begin{abstract}
.
DOI: https://doi.org/10.33262/cienciadigital.v3i4.1.972

On a global level, important transformations in tourism are taking place caused by different social, demographic, environmental, economic, technological and management trends, which will undoubtedly condition the development of tourism in the future. These trends are already manifested in the Ecuadorian Amazon Region. Adapting to the changes, in turn, means for Amazonian tourist destinations, working with a strategic and prospective vision, and it will be necessary for them to know their ability to adapt and react to the changes.

The expectations that have been set in tourism for the development of the province of Pastaza, Ecuador involves addressing the problem of sustainability in order to preserve the natural and cultural heritage, which could be affected by the impacts generated by tourism activities. The objective of the research is to generate a proposal for a system of tourism sustainability indicators for the Pastaza province.
\end{abstract}

This research uses a methodology that combines qualitative and quantitative analysis, part of the conceptualization of tourism and its sustainability and the application of indicators. It proposes 15 indicators for the evaluation of human activities and environmental issues that could evaluate sustainability in areas of tourist use of Pastaza. Indicators, which are

\footnotetext{
${ }^{1}$ Universidad Estatal Amazónica, Ecuador, cliespin@gmail.com

${ }^{2}$ Universidad Estatal Amazónica, Ecuador, eruiz@uea.edu.ec

${ }^{3}$ Universidad de La Habana, Cuba, julianleo2010@gmail.com
} 
characterized by being; methodologically structured, scientifically valid, simple application and disclosure; and, adjustable to the territoriality of different Amazonian tourist destinations with similar characteristics.

Keywords: Tourism, Sustainability, Indicators, Pastaza

\section{Resumen.}

A nivel global, se están produciendo importantes transformaciones en el turismo provocado por diferentes tendencias sociales, demográficas, ambientales, económicas, tecnológicas y de gestión, que sin duda condicionaran el desarrollo del turismo en el futuro. Estas tendencias ya se manifiestan en la región amazónica ecuatoriana. Adaptarse a los cambios, a su vez, supone para los destinos turísticos amazónicos, trabajar con una visión estratégica y prospectiva, y será necesario que previamente conozcan su capacidad de adaptación y reacción ante los cambios.

Las expectativas que se ha cifrado en el turismo para el desarrollo de la provincia de Pastaza, Ecuador implica abordar la problemática de la sostenibilidad en aras de conservar los patrimonios natural y cultural, que podrían ser afectados por los impactos que genera las actividades turísticas. El objetivo de la investigación es desarrollar una propuesta de indicadores vinculados a la sostenibilidad turística para la provincia Pastaza.

Esta investigación emplea una metodología que combina el análisis cualitativo y cuantitativo, parte de la conceptualización del turismo y su sostenibilidad; y, la conformación de una batería de indicadores. Propone 15 indicadores para la evaluación de las actividades humanas y temas ambientales que pudieran medir la sostenibilidad en áreas de uso turístico en Pastaza; Indicadores, que se caracterizan por ser; metodológicamente estructurados, científicamente válidos, de sencilla aplicación y divulgación; y, podrían ser ajustables a la territorialidad de diferentes destinos turísticos amazónicos con similares características.

Palabras claves: Turismo, Sostenibilidad, Indicadores, Pastaza

\section{Introducción.}

El turismo ha ganado importancia a lo largo de las últimas décadas, hasta convertirse en una de las principales actividades económicas a nivel mundial. Para Ecuador el sector turístico es una de las estrategias, para el desarrollo económico, social y protección del patrimonio cultural de las regiones en especial de la amazónica, esta ultima la pobreza aun alcanza el 22\% (Instituto Ecuatoriano de Estadística y Censos INEC, 2017).

La provincia Pastaza se ubica estratégicamente en la zona central de la Amazonía ecuatoriana. Su cabecera cantonal, la ciudad El Puyo, es uno de los accesos más importantes hacia el Oriente ecuatoriano, forma parte de un eje vial que conecta la zona céntrica del país. La Amazonía 
ecuatoriana, gracias a su mega biodiversidad, tiene una posición privilegiada para desarrollar el turismo sostenible. Ecuador está entre los 10 países más biodiversos, se estima que sólo 17 países en el planeta ostentan esta categoría y que estos albergan entre el 60 y el $70 \%$ de la biodiversidad del planeta (National Geographic, 2017).

El desarrollo del turismo en las regiones amazónicas, carece de un sistema de información turística estructurado en base a indicadores de sostenibilidad que permita la toma de decisiones y especialmente la medición de los beneficios y costes en un espacio turístico caracterizado por su mega biodiversidad.

La competitividad y la complementariedad del sector turístico están estrechamente relacionadas con la sostenibilidad de los destinos (en desarrollo y maduros), que dependen principalmente del patrimonio natural y cultural, así como de la territorialidad de la comunidad anfitriona (Sociedad de la Cultura, 2013), (Espin, 2019).

Un sistema de indicadores vinculados al desarrollo sostenible del turismo en la Provincia de Pastaza de la Región Amazónica de Ecuador, aportará conocimientos y herramientas que contribuirán al estudio de la sostenibilidad y vulnerabilidad; pudiendo, ser aplicados a destinos turísticos con características similares.

Como punto de partida para la propuesta de un Sistema de Indicadores para el desarrollo sostenible del turismo (SITS) en la Provincia Pastaza de Ecuador, se realizó el diagnóstico del potencial turístico de la región amazónica de Pastaza (Del Corral \& Rodríguez, 2015). También se llevó a cabo, un estudio en las bases de datos bibliográficos Scopus y Scimago del término "Indicadores de desarrollo sostenible" empleado en títulos y resúmenes y publicados entre 2010 y 2019. Estos aportaron información sobre los posibles tópicos de la investigación (Espin, 2019).

Como regla general, los sistemas de indicadores para el turismo se han basado hasta el presente en un conjunto limitado de variables estadísticas; tales como: el número de visitantes o llegadas, las encuestas laborales y los índices de satisfacción de los visitantes, para llevar a cabo el seguimiento del turismo en sus respectivos destinos (Sancho Pérez \& García Mesanat, 2019).

Estas estadísticas no captan la incidencia del turismo en su totalidad. La recopilación de datos e información sobre una gran variedad de temas relacionados con el efecto que ejerce esta actividad en la economía, la comunidad y el entorno local ayudará a los destinos a obtener una imagen precisa de la realidad.

Trascurridas varias décadas de las primeras definiciones sobre desarrollo sostenible del turismo, la Asamblea General de las Naciones Unidas ha convocado a los países miembros a actuar con fuerza en la implantación de un sistema de indicadores de sostenibilidad para el turismo y la creación de sistemas de información para la sostenibilidad turística (Asamblea General de las Naciones Unidas, 2017). 
El objetivo de esta investigación fue; diseñar un sistema de indicadores turísticos en la provincia Pastaza, que sirva de base para el desarrollo del turismo sostenible y la evaluación de la vulnerabilidad de los destinos turísticos amazónicos, que faciliten su utilización práctica, desde la estructura turística ecuatoriana y los rasgos únicos de Pastaza.

\section{Indicadores vinculados a la Sostenibilidad Turística}

El turismo es en la actualidad un sector fundamental de la economía mundial, en especial en lo que se refiere al comercio internacional de servicios. La gestión del turismo afecta a las condiciones de los destinos y de las comunidades de acogida y, más en general, al futuro de los ecosistemas, las regiones y las naciones. (OMT, 2015, 2018).

Desde principios del decenio de 1990, la OMT ha estado a la cabeza de la elaboración de indicadores de sostenibilidad y de su aplicación al turismo y a los destinos. Durante las dos últimas décadas, se han realizado estudios, eventos científicos y reuniones gubernamentales en diversas regiones del mundo, con el objetivo de crear un sistema de indicadores vinculados a la sostenibilidad turística eficaz para apoyar una mejor toma decisiones en el sector.

Convertir las estadísticas del turismo en una propuesta de valor estratégico para los estados miembros de la Organización Mundial del Turismo "OMT" es una opción. La iniciativa de Medición del Turismo Sostenible es una respuesta a las peticiones persistentes de los países y otras partes interesadas del turismo, para comprender y monitorear el turismo sostenible. La agencia responsable de los indicadores de los Objetivos de Desarrollo Sostenible (ODS) relevantes para el turismo, tiene un papel fundamental que desempeñar en el camino hacia la Agenda 2030 y los 17 Objetivos de Desarrollo Sostenible. (ONU, 2017).

El desconocimiento de los beneficios y costes reales del turismo durante décadas ha sido admitido por diferentes países y organizaciones. Las recomendaciones, elaboradas por el Departamento de Estadísticas de la Cuenta Satélite de Turismo de la OMT, y por la División de Estadísticas de la ONU, proporcionan las bases metodológicas para la recopilación y elaboración de las estadísticas del sector en todos los países/destinos, con independencia del grado de desarrollo de sus sistemas estadísticos. Fueron dirigidas fundamentalmente a las oficinas nacionales de estadística y de las administraciones de turismo que participan en la compilación de estadísticas en base a indicadores vinculados la sostenibilidad. (Cabello, 2013).

La iniciativa de Medición del Turismo Sostenible, la OMT promueve un entendimiento común sobre el fenómeno turístico en relación con otras áreas clave, desde la infraestructura, medio ambiente y hasta las relaciones intrínsecas del tejido social anfitrión, los asuntos sociales, las finanzas. Permitiendo producir datos que sean comparables entre destinos y con otros sectores basados en las realidades territoriales. (OMT, 2015, 2018).

En la $18^{a}$ Reunión del Comité de Estadísticas y Cuenta Satélite de Turismo en la Organización Mundial del Turismo, (2018) reiteró una vez más la necesidad de ampliar las estadísticas del 
turismo más allá de un enfoque económico. Así, como para incluir aspectos sociales y ambientales con este propósito se aprobó la iniciativa "Medición del Turismo Sostenible para mejorar la toma de decisiones". La cual ha recibido el más alto nivel de aprobación y apoyo por parte de la Asamblea General de la OMT y la Comisión de Estadísticas de la Organización de Naciones Unidas (ONU).

Tabla 1: Principales acciones sobre de indicadores vinculados a la sostenibilidad turística.

\begin{tabular}{|c|c|c|}
\hline Año & Institución/eventos & Acción \\
\hline 1991 & $\begin{array}{l}\text { Organización Mundial del turismo, } \\
\text { OMT }\end{array}$ & $\begin{array}{l}\text { Iniciativa para desarrollar Indicadores de } \\
\text { turismo sostenible de uso global. }\end{array}$ \\
\hline 1992 & Cumbre Mundial de Río de Janeiro. & $\begin{array}{c}\text { El desarrollo sostenible del turismo. } \\
\text { Principios del programa de acción "Agenda } \\
21 \text { ". }\end{array}$ \\
\hline 1995 & $\begin{array}{c}\text { Organización Mundial del turismo, } \\
\text { OMT }\end{array}$ & $\begin{array}{c}\text { Guía práctica para el desarrollo y uso de } \\
\text { indicadores de turismo sostenible, a partir de } \\
\text { las cuales se logró desarrollar un grupo de } \\
\text { indicadores clave. }\end{array}$ \\
\hline 1996 & $\begin{array}{l}\text { OMT, World Travel Tourism Council } \\
\text { (WTTC) y Earth Council (EC) }\end{array}$ & $\begin{array}{l}\text { Agenda } 21 \text { para el sector de turismo } \\
\text { sostenible y Viajes. }\end{array}$ \\
\hline 2004 & $\begin{array}{c}\text { Organización Mundial del turismo, } \\
\text { OMT }\end{array}$ & $\begin{array}{c}\text { Indicadores de Desarrollo Sostenible para } \\
\text { Destinos Turísticos. }\end{array}$ \\
\hline 2005 & $\begin{array}{c}\text { OMT/PNUMA y el Programa de las } \\
\text { Naciones Unidas para el Medio } \\
\text { Ambiente }\end{array}$ & $\begin{array}{c}\text { Making Tourism more sustainable / } \\
\text { Indicadores de desarrollo sostenible para } \\
\text { destinos turísticos. }\end{array}$ \\
\hline 2010 & $\begin{array}{c}\text { OMT y el Consejo Global de Turismo } \\
\text { Sostenible (GSTC) }\end{array}$ & $\begin{array}{c}\text { El GSTC se constituyó oficialmente en } 2010 \\
\text { como organismo para la creación y la gestión } \\
\text { de normas para el turismo sostenible. }\end{array}$ \\
\hline 2012 & Conferencia de Rio +20 & $\begin{array}{l}\text { Asegurar un compromiso político renovado } \\
\text { con el desarrollo sostenible, evaluar los } \\
\text { progresos realizados y las brechas que aún } \\
\text { existen. }\end{array}$ \\
\hline 2015 & $\begin{array}{l}\text { OMT. «Hacia un marco estadístico para } \\
\text { la medición del turismo sostenible» }\end{array}$ & $\begin{array}{l}\text { Primer paso hacia el desarrollo de } \\
\text { información estadística básica para las } \\
\text { regiones y otros agregados territoriales } \\
\text { subnacionales: (SIT-R) }\end{array}$ \\
\hline 2016 & $\begin{array}{c}\text { OMT, segunda reunión de expertos en } \\
\text { Medición del Turismo Sostenible en } \\
\text { Madrid. }\end{array}$ & $\begin{array}{c}\text { El trabajo de este grupo de expertos es clave } \\
\text { en el marco de la contabilidad } \\
\text { medioambiental aplicada al turismo. }\end{array}$ \\
\hline
\end{tabular}


2017 Medición del Turismo Sostenible Sexta Conferencia Internacional de la OMT sobre Estadísticas del Turismo

$201818^{\text {a }}$ Reunión del Comité de Estadísticas

y Cuenta Satélite de Turismo en la OMT
Apoyada por la División de Estadística de la ONU, la iniciativa de la OMT «Hacia un marco estadístico para medir el turismo sostenible»

La OMT encabeza el progreso hacia un enfoque estadístico consistente y coherente para medir el turismo sostenible vinculado indicadores.

Fuente: Elaboración propia.

\section{Metodología}

Durante la revisión bibliográfica se identificaron, principalmente, manuales, artículos científicos y trabajos de consultoría, que contribuyeron ampliamente a la consecución de los objetivos planteados con anterioridad. La búsqueda se realizó a través de, centros de documentación y datos. Al final del proceso se habían utilizado más de 1600 referencias.

A partir de un proceso inicial de reflexión y revisión de la temática en investigación, se pasa a la definición de criterios y al diseño del sistema de indicadores turísticos, para posteriormente realizar la validación mediante técnicas de participación pública discusiones de grupo, Método Delphi y métodos de optimización Multicriterio. El proceso comenzó con la definición de los problemas a abordar y la selección de los expertos sobre el desarrollo sostenible del turístico, posteriormente se procedió a establecer los objetivos de la investigación, la formulación de los ejes de discusión y la preparación de los cuestionarios (Reguant-Álvarez \& Torrado-Fonseca, 2016); (Blasco-Blasco \& Coll Serrano, 2016)

Figura 1: Planteamiento metodológico

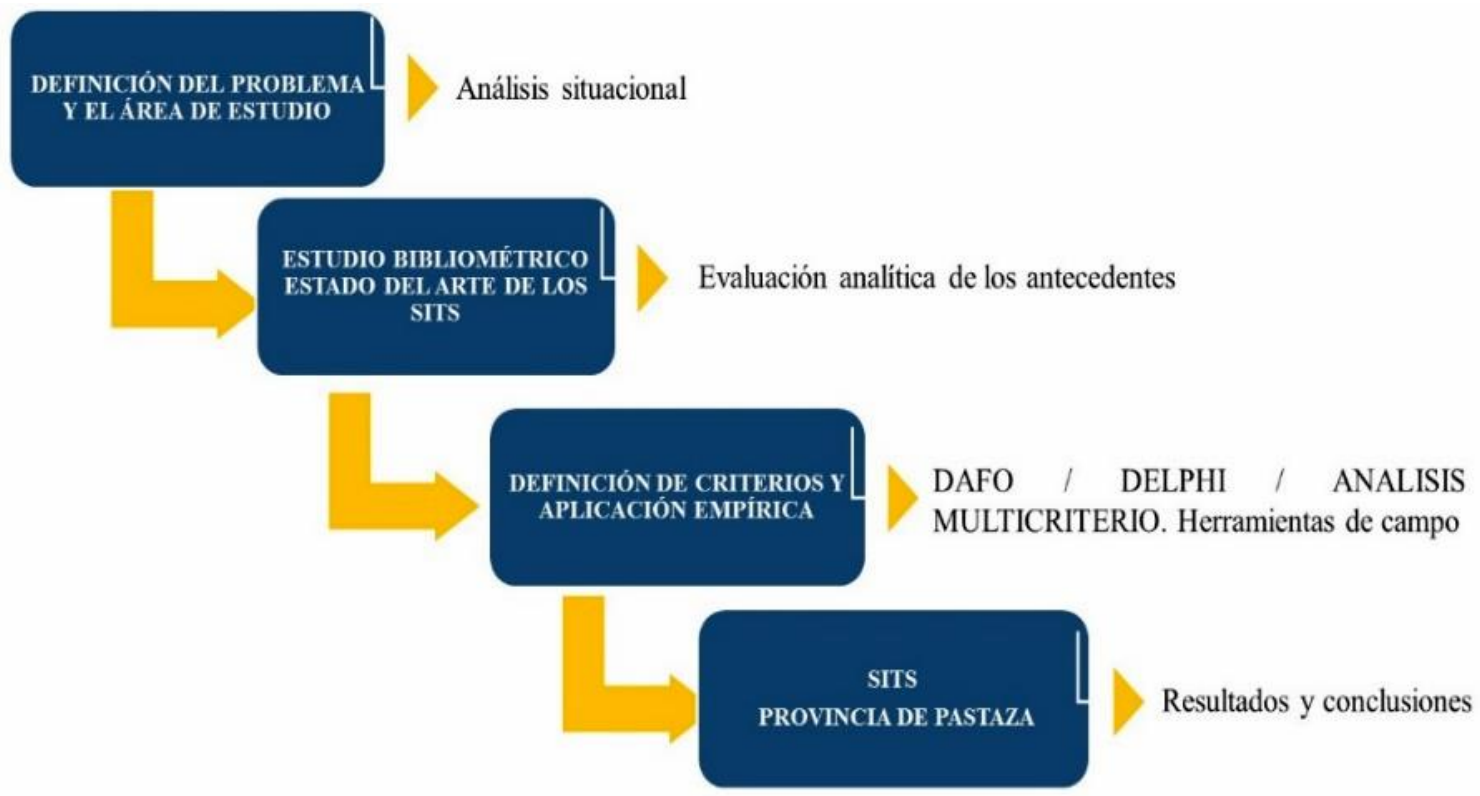

Fuente: Elaboración propia 
Las encuestas y entrevistas a los diferentes actores del turismo en Pastaza, se estructuró en base a 35 preguntas, sobre el desarrollo de las actividades turísticas, que finalmente fueron agrupadas en los siguientes aspectos:

1. Obstáculos más importantes

2. Entorno socio-económico

3. Medio Natural

4. Infraestructuras, equipamientos y servicios.

5. Recursos humanos y socioculturales.

6. Recursos financieros.

7. Planeación estratégica.

8. Otros

Tabla 2. Directivos de Gobierno y Organismos entrevistados

\begin{tabular}{ll}
\hline \multicolumn{1}{c}{ Institución } & \multicolumn{1}{c}{ Funcionario / Cargo } \\
\hline Ministerio del Turismo & Lcda. Balvanera Cruz / Coordinadora Zonal Tres de \\
MINTUR & Turismo (Pastaza, Tungurahua, Chimborazo y Cotopaxi). \\
Ministerio del Turismo & MSC. Nelly Duran / directora de la Unidad Provincia de \\
MINTUR & Pastaza \\
GAD Provincial de Pastaza & MSC. Víctor Merino Arcos / Promotor Comunitario \\
GAD Municipal de Pastaza & MCS. Judith Cachipuendo Castillo / Directora \\
& Departamento de Turismo \\
GAD Municipal de Arajuno & Lic. Efrén Calapucha / Director Municipal de Desarrollo \\
& Cantonal \\
GAD Municipal de Mera & Lic. Maritza Villarroel / Técnica de Turismo \\
GAD Municipal de Santa Clara & Ing. Cesar Castro / Alcalde \\
Ministerio del Ambiente & Ing. Angélica Navarrete / Directora Provincial \\
Cámara de Comercio de Pastaza & Lic. Roney Sancho / Presidente \\
Mueso Etno-arqueológico de & Ing. Edwin Sánchez / Director \\
Pastaza & \\
Cámara de Turismo de Pastaza & Sr. Juan Carlos Freire / Presidente \\
\hline
\end{tabular}

Fuente: Elaboración propia

\section{Análisis DAFO}

Se realizó un taller para el análisis del desarrollo turístico de Pastaza el método utilizado fue el diagnóstico estratégico participativo ponderado (DEPP), desarrollado por (Mielke \& Sperandio, 2009), que consiste en la fusión de dos métodos tradicionales de diagnóstico: el DAFO (Deficiencias, Amenazas, Fuerzas y Oportunidades) y la técnica Delhi, con lo cual se obtienen las ventajas las que ofrecen las metodologías DAFO y Delphi. El estudio tiene carácter 
multidisciplinario con la evaluación cuantitativa del proceso de desarrollo de la provincia Pastaza como destino turístico (Del Corral \& Rodríguez, 2015).

\section{Selección de los Expertos}

La selección de los expertos para la realización del estudio Delphi se estableció, como criterio fundamental, por el grupo coordinador. Los expertos que participaron en la selección de los indicadores fueron escogidos por su especialización en temas de desarrollo sostenible del turismo y proceden tanto de ámbitos de la investigación como de la planificación y gestión del sector.

La versión del cuestionario consensuada por los miembros del grupo se sometió a la validación de expertos seleccionados, con la intención de recabar los criterios cuantitativos y cualitativos. El envío y la recepción del cuestionario se realizaron por correo electrónico con una breve introducción al tema, la explicación del objetivo y el método que se utiliza en la investigación.

En base a la experiencia contrastada en la literatura revisada para este trabajo, se estableció que el grupo de 15 expertos que debería participar en este análisis Delphi estuviera formado por:

1. Profesores universitarios vinculados con la investigación y la planificación turística de los espacios naturales, o de las relaciones entre turismo, territorio y medio ambiente.

2. Profesionales de las administraciones públicas ecuatorianas, turísticas y medio ambientales.

3. Miembros de instituciones y de organismos, públicos y privados; relacionados con la planificación y gestión de espacios naturales.

4. Profesionales de empresas privadas con experiencia en planificación y gestión turística de espacios naturales.

5. En todo caso, se estableció como requisito la existencia de una estrecha vinculación entre la actividad profesional, institucional y/o investigadora de los expertos invitados a colaborar y los temas objeto de estudio.

Tabla 3. Número de expertos en cada una de las áreas del conocimiento

\begin{tabular}{|c|c|c|c|}
\hline $\begin{array}{c}\text { No. } \\
\text { Expertos }\end{array}$ & Áreas de competencia & $\begin{array}{c}\text { No. } \\
\text { Expertos }\end{array}$ & Áreas de conocimiento \\
\hline 8 & Gestión turística local y estatal. & 7 & Ciencias políticas y Economía urbana. \\
\hline 5 & $\begin{array}{c}\text { Gestión del Patrimonio Cultural } \\
\text { y la Historia. }\end{array}$ & 3 & $\begin{array}{l}\text { Sociología urbana - gestión de } \\
\text { asentamientos humanos. }\end{array}$ \\
\hline 5 & $\begin{array}{c}\text { Agrupaciones de prestadores de } \\
\text { servicios turísticos. }\end{array}$ & 10 & $\begin{array}{c}\text { Planificación integral para el desarrollo } \\
\text { del turismo - patrimonio. }\end{array}$ \\
\hline 12 & $\begin{array}{l}\text { Sector académico Planificación } \\
\text { de la gestión turística. }\end{array}$ & 9 & Turismo - planificación ambiental. \\
\hline 6 & Planificación urbana y regional. & 10 & Desarrollo sostenible. \\
\hline
\end{tabular}

Fuente: Elaboración propia 
Figura 2: Coeficiente de competencia de los expertos seleccionados

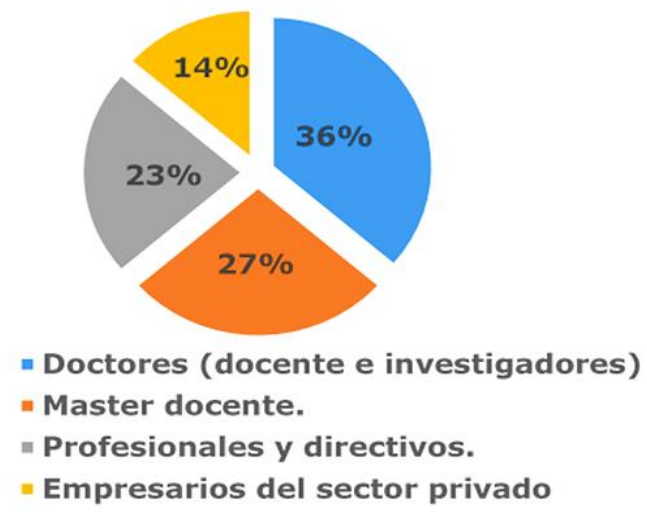

Fuente: Elaboración propia

K: Coeficiente de Competencia $=0,90$

Kc: Coeficiente de Conocimiento=0,91

Ka: Coeficiente de Argumentación=0,89

\section{DELPHI}

\section{Coeficiente de Competencia}

$\mathrm{K}=0,5(\mathrm{Kc}+\mathrm{Ka})$

$0,8<\mathrm{K}<1,0$ Alto

$0,5<\mathrm{K}<0,8$ Medio

$\mathrm{K}<0,5$ Bajo

15 expertos con experiencia promedio de 25 años.

\section{Resultados}

En los últimos años, se asiste a un amplio desarrollo en el ámbito de los indicadores para la toma de decisiones, particularmente por parte de los países industrializados y de algunas organizaciones internacionales, pero también en varios países de Latinoamérica, se han presentado iniciativas que comprenden diversos parámetros de medición y enfoques metodológicos. La sostenibilidad ambiental recibió la mayor atención, pero más recientemente el enfoque de desarrollo sostenible, incorporando las dimensiones económica, social, ambiental e institucional.

La propuesta del Sistema de Indicadores para el Turismo Sostenible (SITS), se ha desarrollado a partir del Diagnóstico Estratégico Participativo Ponderado (DEPP). En la consulta y las encuestas participaron 37 expertos, quienes emitieron criterios en las diferentes etapas de selección de los indicadores. Esto reduce la posible subjetividad en el proceso de investigación. El SITS incluyó las cuatro dimensiones del desarrollo sostenible propuestas por la Comisión de Desarrollo Sostenible de las Naciones Unidas en la definición conceptual: social, económica, institucional y ambiental. 
Tabla 4. Indicadores vinculados a la sostenibilidad turística propuesto a nivel internacional

\begin{tabular}{ccc}
\hline Organismos & $\begin{array}{c}\text { Año / } \\
\text { creación }\end{array}$ & $\begin{array}{c}\mathbf{N}^{\circ} \text { de } \\
\text { indicadores }\end{array}$ \\
\hline $\begin{array}{cc}\text { United Nations - Commission Sustainable Development, UN } \\
\text { Organization for economic co-operation and development, OECD }\end{array}$ & 1996 & 134 \\
National Indicators and Reporting Office of Environment Canada. & 2003 & 53 \\
Colombia - Ministerio del Medio Ambiente & 2003 & 55 \\
Organización Mundial del Turismo, Indicadores de desarrollo & 2004 & 33 \\
sostenible para los destinos turísticos. & 58 \\
Sistema Español de Indicadores Ambientales de Turismo & 2003 & 63 \\
Argentina - Ministerio de Salud y Ambiente de la Nación & 2005 & 65 \\
Red de redes de desarrollo local sostenible & 2010 & 30 \\
Sistema Europeo de Indicadores Turísticos & $2013 / 2016$ & 67 \\
Comisión de Turismo del Caribe, CTO & 2014 & 24 \\
Autoridad ambiental, Panamá & 2016 & 20 \\
Investigadores & 2017 & 153 \\
& Total & $\mathbf{7 5 5}$ \\
\hline
\end{tabular}

Fuente: Elaboración propia

A partir de los indicadores reportados internacionalmente para medir la sostenibilidad turística se realizó un proceso de selección basado en el consenso del grupo coordinador y expertos que participaron en el estudio DAFO, se decidió la consulta por la técnica Delphi de 38 indicadores (tablas 4 y 5). De la propuesta de indicadores, siete recibieron calificaciones inferiores a 4 puntos, escala empleada tipo Likert (tabla 6).

Tabla 5. Indicadores vinculados a la sostenibilidad turística, Actividades Humanas

\begin{tabular}{ccccc}
\hline Tema & No. & Indicadores & Promedio & $\begin{array}{c}\text { Deviación } \\
\text { Típica }\end{array}$ \\
\hline $\begin{array}{c}\text { Producción y } \\
\text { patrones de } \\
\text { consumo }\end{array}$ & 1 & Uso de productos químicos dañinos a la & 3,9 & 0,316 \\
& 2 & $\begin{array}{c}\text { salud o al ambiente. } \\
\text { planificación territorial o desarrollo que } \\
\text { incluya el turismo. }\end{array}$ & 4,6 & 0,516 \\
& 3 & $\begin{array}{c}\text { Uso continuo de la evaluación de impacto } \\
\text { ambiental para el turismo. }\end{array}$ & 5 & 0,000 \\
& 4 & $\begin{array}{c}\text { Ingreso de turistas y de divisas, tasa de } \\
\text { crecimiento anual. }\end{array}$ & 4 & 0,943 \\
& 5 & $\begin{array}{c}\text { Llegada de turistas (distribución a lo largo } \\
\text { del año). }\end{array}$ & 4,8 & 0,422 \\
\hline
\end{tabular}




\begin{tabular}{|c|c|c|c|c|}
\hline $\begin{array}{l}\text { Estructura } \\
\text { económica }\end{array}$ & 7 & $\begin{array}{c}\text { Índice de ingresos propios por la } \\
\text { actividad. }\end{array}$ & 4,4 & 0,516 \\
\hline Vivienda & 8 & Suelo artifializado. & 4,2 & 1,033 \\
\hline \multirow{4}{*}{ Educación } & 9 & $\begin{array}{l}\text { Relación entre graduados como } \\
\text { profesionales: hombres y mujeres. }\end{array}$ & 3,00 & 0,000 \\
\hline & 10 & $\begin{array}{c}\text { Información dada a los visitantes antes y } \\
\text { durante las visitas. }\end{array}$ & 3,4 & 0,516 \\
\hline & 11 & $\begin{array}{l}\text { Tipo y cantidad de entrenamiento dados a } \\
\text { los empleados del turismo. }\end{array}$ & 4,4 & 0,516 \\
\hline & 12 & $\begin{array}{c}\text { Tasa de alfabetismo de los adultos de la } \\
\text { zona. }\end{array}$ & 3,00 & 0,943 \\
\hline \multirow{3}{*}{ Seguridad } & 13 & Número de incidentes. & 3,2 & 0,632 \\
\hline & 14 & Tasa de criminalidad. & 4,8 & 0,422 \\
\hline & 15 & Cociente del salario femenino. & 4 & 0,000 \\
\hline \multirow[t]{4}{*}{ Empleo } & 16 & $\begin{array}{l}\text { Medio al salario masculino (Equidad de } \\
\text { género). }\end{array}$ & 4,2 & 0,422 \\
\hline & 17 & Índice de desempleo. & 5 & 0,000 \\
\hline & 18 & Tensión entre visitantes y anfitriones. & 4,8 & 0,422 \\
\hline & 19 & $\begin{array}{c}\text { Implicación de los residentes en las } \\
\text { actividades turísticas. }\end{array}$ & 4,4 & 0,516 \\
\hline \multirow[t]{2}{*}{ Social / cultural } & 20 & $\begin{array}{l}\text { Satisfacción de la comunidad anfitriona } \\
\text { hacia el desarrollo del turismo. }\end{array}$ & 4,9 & 0,316 \\
\hline & 21 & $\begin{array}{c}\text { Continuación de actividades tradicionales } \\
\text { de los residentes locales. }\end{array}$ & 4,9 & 0,316 \\
\hline \multirow{3}{*}{$\begin{array}{c}\text { Dinámica } \\
\text { demográfica }\end{array}$} & 22 & Tasa de crecimiento de la población. & 3 & 0,000 \\
\hline & 23 & Densidad de la población. & 3,8 & 0,422 \\
\hline & 24 & Establecimientos públicos y capacidad. & 4,1 & 0,316 \\
\hline \multirow{2}{*}{ Salud } & 25 & Esperanza de vida al nacer. & 3,1 & 0,316 \\
\hline & 26 & $\begin{array}{l}\text { Salud de la comunidad (de la política } \\
\text { sanitaria relacionada con el turismo). }\end{array}$ & 4,4 & 0,516 \\
\hline
\end{tabular}

Fuente: Elaboración propia

Tabla 6. Propuesta de indicadores para la sostenibilidad turística, Temas Ambientales

\begin{tabular}{ccccc}
\hline \multirow{2}{*}{ Temas } & \multirow{2}{*}{ No. } & Indicadores & \multirow{2}{*}{ Promedio } & Desviación \\
Típica
\end{tabular}




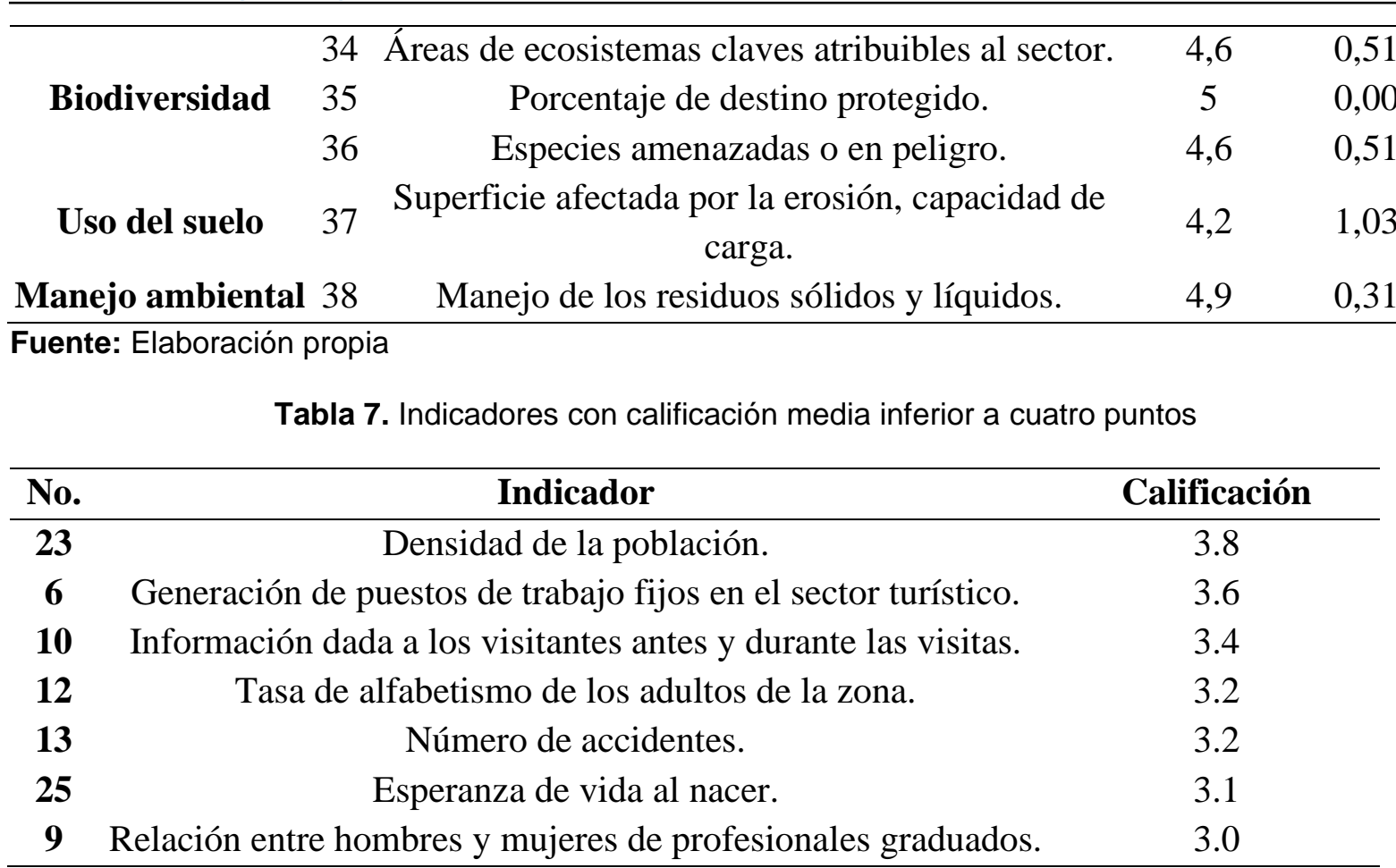

Fuente: Elaboración propia

Los indicadores con calificación media inferior a cuatro puntos corresponden a los evaluados para las actividades humanas; mientras que los indicadores evaluados para temas ambientales alcanzaron mayor prioridad. Lo que confirma la relevancia que se atribuye al potencial del patrimonio natural y cultural de la Amazonia y el significado de su puesta en valor para la actividad turística.

\section{Consulta a terceros para la validación de la propuesta de indicadores vinculados al turismo sostenible en Pastaza}

Las calificaciones otorgadas por cada uno de los evaluadores se analizaron para cada una de las cinco preguntas. Se determinó la desviación típica o estándar, lo que permitió establecer el consenso por parte de los evaluadores al considerar el Sistema de Indicadores propuesto como un instrumento idóneo para medir la sostenibilidad de las áreas con uso turístico.

Tabla 8. Cuestionario de evaluación para la validación, propuesta de Indicadores vinculados a la Sostenibilidad turística en Pastaza

\begin{tabular}{|c|c|c|c|c|}
\hline No & Definición & Media & $\begin{array}{c}\text { Desviación } \\
\text { Típica }\end{array}$ & Resultado \\
\hline 1 & $\begin{array}{l}\text { El Sistema de indicadores propuesto es } \\
\text { coherente con la evaluación } \\
\text { conceptual que pretende cuantificar. } \\
\text { (medir la sostenibilidad de las áreas } \\
\text { con uso turístico) }\end{array}$ & 4,8 & 0,422 & $\begin{array}{l}\text { (4) Más bien de } \\
\text { acuerdo } \\
\text { (5) Totalmente de } \\
\text { acuerdo. }\end{array}$ \\
\hline
\end{tabular}


El sistema de indicadores propuesto es

2 de utilidad práctica para el objetivo que se persigue.

Considera que no existe duplicidad o

3 solapamiento entre los indicadores propuestos por el sistema.

Todos los indicadores propuestos en el sistema son relevantes.

Los indicadores propuestos son

5
$3,7 \quad 0,483$

$4,7 \quad 0,483$

$4,9 \quad 0,316$

4,7

0,483 sostenibilidad de las áreas con uso turístico en el cantón Pastaza.

(4) Más bien de acuerdo

(5) Totalmente de acuerdo

(4) Más bien de acuerdo

(5) Totalmente de acuerdo

(3) Indiferente

(4) Más bien de acuerdo

(4) Más bien de acuerdo

(5) Totalmente de acuerdo

Fuente: Elaboración propia

\section{Índice sintético de operatividad relativa (IOR)}

En base a las valoraciones de los expertos sobre el sistema propuesto de indicadores de sostenibilidad para áreas con uso turístico en Pastaza, se construyó un índice sintético de operatividad relativa (IOR). Los expertos no recibieron información sobre las calificaciones finales del estudio Delphi, A partir de las puntuaciones de relevancia, efectividad y factibilidad otorgadas por los mismos (Coll-Serrano, Blasco-Blasco, Carrasco-Arroyo, \& Vila-Lladosa, 2013). Se consideró la formulación general IOR:

\section{$\operatorname{IOR}(\mathbf{I j})=(w R \mathbf{R j}+w E \mathbf{E j}+w F \mathbf{F j})$}

Dónde: Rj, Ej y Fj representan las puntuaciones medianas obtenidas por el indicador j con respecto a los criterios de relevancia, especificad y factibilidad. $\mathrm{wR}, \mathrm{wE}$ y wF denotan las ponderaciones o pesos respectivos de cada criterio en el índice (Grandzol; Gershon, 1994; Tague, 2004), que inicialmente han sido prefijadas a los valores de las puntuaciones dadas por los expertos $(\mathrm{wR}=0,5$; $\mathrm{wE}=0,25 \mathrm{y} w \mathrm{~F}=0,25)$.

- $\quad$ Relevancia. - Grado de importancia y utilidad que tiene el indicador para medir potencialmente el fenómeno observado, posibilitando la toma de decisiones por parte del gestor.

- $\quad$ Especificidad. - En qué medida la variación del indicador responde efectivamente a variaciones del fenómeno que se desea monitorizar y minimiza el efecto de factores exógenos en su variación.

- $\quad$ Factibilidad. - Posibilidad de fijar un valor deseado para el indicador, es decir, en qué medida es posible asignar un objetivo cuantificable basado en el indicador evaluado. 
Los 31 indicadores seleccionados mediante el estudio Delphi y la consulta a terceros fueron evaluados mediante la aplicación del IOR, de ellos 15 recibieron calificaciones superiores.

Tabla 9. Indicadores seleccionados a partir del índice sintético de operatividad relativa IOR, Actividades Humanas

\begin{tabular}{|c|c|c|c|c|c|c|}
\hline \multicolumn{7}{|c|}{ Categoría / Actividades Humanas } \\
\hline Tema & No. & Indicadores & $\mathbf{W r}+\mathbf{R j}$ & $\mathbf{W r}+\mathbf{E j}$ & $\mathbf{W r}+\mathbf{F j}$ & IOR \\
\hline \multirow[t]{4}{*}{ Turismo } & 1 & $\begin{array}{l}\text { Existencia de algún proceso de } \\
\text { planificación territorial o de } \\
\text { desarrollo que incluya el } \\
\text { turismo. }\end{array}$ & 2,5 & 1,25 & 1,13 & 4,88 \\
\hline & 2 & $\begin{array}{l}\text { Uso continuo de la evaluación } \\
\text { de impacto ambiental para el } \\
\text { turismo. }\end{array}$ & 2,5 & 1,25 & 1,25 & 5,00 \\
\hline & 3 & $\begin{array}{l}\text { Ingreso de turistas y de divisas, } \\
\text { tasa de crecimiento anual. }\end{array}$ & 2,5 & 1,25 & 1,00 & 4,75 \\
\hline & 4 & $\begin{array}{l}\text { Llegada de turistas (distribución } \\
\text { a lo largo del año). }\end{array}$ & 2,5 & 1,25 & 1,00 & 4,75 \\
\hline \multirow[t]{3}{*}{ Social / Cultural } & 5 & $\begin{array}{l}\text { Implicación de los residentes en } \\
\text { las actividades turísticas. }\end{array}$ & 2,5 & 1,25 & 1,13 & 4,88 \\
\hline & 6 & $\begin{array}{l}\text { Satisfacción de la comunidad } \\
\text { anfitriona hacia el desarrollo del } \\
\text { turismo. }\end{array}$ & 2,5 & 1,25 & 1,13 & 4,88 \\
\hline & 7 & $\begin{array}{l}\text { Continuación de actividades } \\
\text { tradicionales de los residentes } \\
\text { locales. }\end{array}$ & 2,5 & 1,25 & 1,25 & 5,00 \\
\hline
\end{tabular}

Fuente: Elaboración propia

La selección de indicadores se determinó no sólo en el valor del IOR sino también, específicamente, en las puntuaciones asignadas por los expertos con respecto al criterio de factibilidad. Establecimiento de un valor mínimo o umbral de factibilidad diferente por actuación prioritaria que delimita qué indicadores son los más factibles.

El umbral de factibilidad, que es específico para cada actuación prioritaria, se define de manera que permita retener al menos dos indicadores entre los correspondientes a cada actuación prioritaria 
Tabla 10. Indicadores seleccionados a partir del índice sintético de operatividad relativa IOR, Temas Ambientales.

\begin{tabular}{|c|c|c|c|c|c|c|}
\hline \multicolumn{7}{|c|}{ Categoría / Temas Ambientales } \\
\hline Tema & & Indicadores & $\mathbf{W r}+\mathbf{R j}$ & $\mathbf{W r}+\mathbf{E j}$ & $\mathbf{W r}+\mathbf{F j}$ & IOR \\
\hline $\begin{array}{l}\text { Manejo de } \\
\text { ecosistemas }\end{array}$ & 8 & $\begin{array}{c}\text { Ecosistemas bajo protección o } \\
\text { manejo ambiental. }\end{array}$ & 2,5 & 1,25 & 1,25 & 5,00 \\
\hline Aire & 9 & $\begin{array}{l}\text { Políticas de manejo de la } \\
\text { polución del aire. }\end{array}$ & 2,5 & 1,25 & 1 & 4,75 \\
\hline \multirow[t]{2}{*}{ Agua } & 10 & Cobertura de agua potable. & 2,5 & 1,25 & 1,25 & 5,00 \\
\hline & 11 & $\begin{array}{l}\text { Cobertura de tratamiento de } \\
\text { aguas residuales. }\end{array}$ & 2,5 & 1,25 & 1,25 & 5,00 \\
\hline \multirow[t]{3}{*}{ Biodiversidad } & 12 & $\begin{array}{c}\text { Áreas de ecosistemas clave } \\
\text { atribuibles al sector. }\end{array}$ & 2,5 & 1,25 & 1 & 4,75 \\
\hline & 13 & $\begin{array}{l}\text { Porcentaje de destino } \\
\text { protegido. }\end{array}$ & 2,5 & 1,25 & 1,25 & 5,00 \\
\hline & 14 & $\begin{array}{l}\text { Especies amenazadas o en } \\
\text { peligro. }\end{array}$ & 2,5 & 1,25 & 1,125 & 4,88 \\
\hline $\begin{array}{c}\text { Manejo } \\
\text { ambiental }\end{array}$ & 15 & $\begin{array}{c}\text { Manejo de los residuos sólidos } \\
\text { y líquidos. }\end{array}$ & 2,5 & 1,25 & 1,25 & 5,00 \\
\hline
\end{tabular}

Fuente: Elaboración propia

\section{Conclusiones.}

- La selección de los indicadores de sostenibilidad se realizó a partir de los propuestos a nivel internacional. La propuesta de sistema de indicadores para el turismo sostenible (SITS), para áreas con uso turístico en Pastaza, se diseñó a partir del Diagnóstico Estratégico Participativo Ponderado del Turismo (DEPP) y la consulta a 37 expertos quienes participaron en la selección de los indicadores, lo cual reduce la posible subjetividad en el proceso de la investigación.

- Posteriormente se analizaron las calificaciones otorgadas por cada uno de los evaluadores y se determinó la desviación típica o estándar. Esto permitió establecer el consenso por parte de los evaluadores al considerar el sistema de indicadores propuesto como un instrumento idóneo para medir la sostenibilidad de las áreas naturales con uso turístico.

- En base a las valoraciones de los expertos sobre sistema de indicadores de sostenibilidad para áreas con uso turístico en la provincia Pastaza, se construyó un índice sintético de operatividad relativa (IOR). Esta herramienta considera la relevancia, la efectividad y la factibilidad de cada uno de los indicadores. Como resultado final, se presenta un total de 15 indicadores; de los cuales, siete corresponden a las actividades humanas y ocho a temas ambientales.

- La propuesta indicadores vinculados a la sostenibilidad turística en Pastaza, puede contribuir a fomentar el turismo sostenible y la evaluación de vulnerabilidad del destino, facilitando su utilización práctica desde la estructura turística ecuatoriana y los rasgos distintivos de Pastaza, con especial atención a la mega diversidad de sus patrimonios. 


\section{Referencias bibliográficas.}

Asamblea General de las Naciones Unidas. (21 - 23 de June de 2017). Measuring Sustainable Tourism: A Call for Action - Report of the 6th International Conference on Tourism Statistics, Manila, Philippines, No of Pages: 50. Obtenido de ISBN 978-92-844-1895-4, Digital; ISBN 978-92-844-1894-7, Impreso.: http://statistics.unwto.org/fr/node/48735

Blasco-Blasco, O., \& Coll Serrano, V. (2016). Selección de indicadores basada en métodos de optimización multicriterio una aplicación a la estrategia de cultura y desarrollo de España. Obtenido de Rect@: Revista Electrónica de Comunicaciones y Trabajos de ASEPUMA, Vol. 17, No. 12, págs. 19-33: https://dialnet.unirioja.es/servlet/articulo?codigo=6466464

Cabello, J. (2013). Synthetic indicators of mutual funds' environmental responsibility: 236, 313 325. Obtenido de An application of the Reference Point Method. European Journal of Operational Research: http://www.sciencedirect.com/science/article/pii/S03772

Camacho-Rui, E., \& Carrillo, A. (2016). Indicadores de sostenibilidad para el ecoturismo en Méxic. Recuperado el marzo de 2017, de Estado actual: http://www.scielo.org.mx/scielo.php?script=sci_arttext\&pid=S1665$80272016000100011 \& \operatorname{lng}=$ es\&tlng=es.

el Corral, V. H., \& Rodríguez, J. L. (2015). Desarrollo turístico de Pastaza: diagnóstico estratégico participativo ponderado. Puyo: ABET, Juiz de FORA, 35.

Espin, C. (2019). Sistema de indicadores para el Turismo Sostenible, Pastaza; Tesis Doctoral Universidad de La Habana. La Habana, Cuba.

Hechavarria, L. (2016). Best practices in tourist destinations sustainable development assessment: a literature review. Turismo. Estudos\&Práticas (RTEP/UERN), Mossoró/RN, vol. 5, n. 2, 149-185 (Vol. vol. 5).

nstituto Ecuatoriano de Estadística y Censos INEC. (3 de junio de 2017). Instituto Ecuatoriano de Estadística y Censos. Obtenido de http://www.ecuadorencifras.gob.ec/estadisticas/

National Geographic. (25 de marzo de 2017). National Geographic. Obtenido de http://www.nationalgeographic.com.es/

Reguant-Álvarez, M., \& Torrado-Fonseca, M. (2016). El método Delphi . REIRE Revista d'Innovación, DOI: 10.1344/reire2016.9.1916, 87-102.

odriguez, D., \& Alavarez, J. (2013). Vulnerabilidad del turismo y comunicación institucional ante desastres. Estudio de caso, 15.

Sancho Pérez, A., \& García Mesanat, G. (29 de Mayo de 2019). ¿Que indica un indicador? Análisis comparativo en los destinos turísticos. Obtenido de Revista de análisis turístico, ISSN 2254-0644.: https://aecit.org/jornal/index.php/AECIT/article/view/90 
Sociedad de la Cultura. (2013). Cultura y Turismo: Factores del desarrollo económico y social. Buenos Aires, Argentina.: Secretaria de Cultura de la presidencia de la Nación. Obtenido de https://issuu.com/secretariadecultura/docs/cultura_y_turismo 
PARA CITAR EL ARTÍCULO INDEXADO.

Esoín Ortíz, C. G., Ruiz, E. E., \& Rodríguez Rodríguez, J. L. (2019). Propuesta, indicadores vinculados a la sostenibilidad turística en Pastaza, Ecuador. Ciencia Digital, 3(4.1), 21-38. https://doi.org/10.33262/cienciadigital.v3i4.1.972

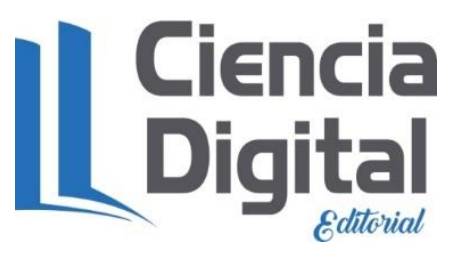

El artículo que se publica es de exclusiva responsabilidad de los autores y no necesariamente reflejan el pensamiento de la Revista Ciencia Digital.

El artículo queda en propiedad de la revista y, por tanto, su publicación parcial y/o total en otro medio tiene que ser autorizado por el director de la Revista Ciencia Digital.
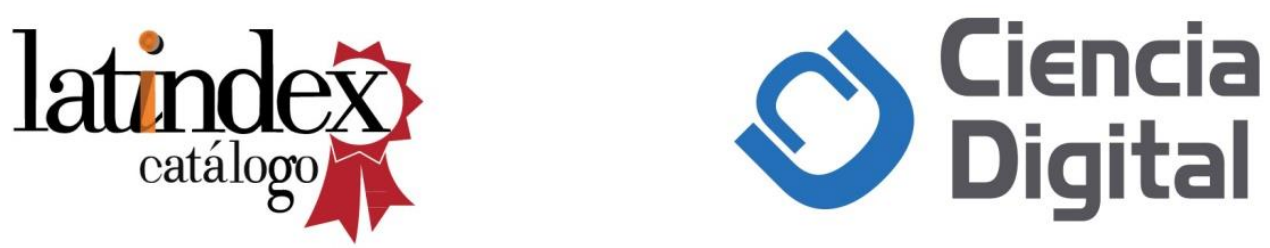